\title{
Maximum joint torque dependency of the crawl swimming with optimized arm stroke
}

\author{
Motomu NAKASHIMA* and Ayako ONO* \\ * Graduate School of Science and Engineering, Tokyo Institute of Technology \\ 2-12-2 Oookayama, Meguro-ku, Tokyo 152-8552, Japan \\ E-mail: motomu@mech.titech.ac.jp
}

Received 2 April 2013

\begin{abstract}
The objectives of this study were to solve computationally the arm stroke in the crawl swimming which maximized the swimming speed for different maximum joint torque conditions, and to investigate the maximum joint torque dependency of the crawl swimming with the optimized arm stroke. In the optimizing calculation, the swimming human simulation model, SWUM, was used for the simulation of the crawl swimming. The maximum joint torque characteristics, which were constructed in the previous study, were imposed as the constraint condition. In order to investigate the maximum joint torque dependency of the optimized arm stroke, four levels of maximum joint torque were prepared. The following findings were obtained from the results of optimization: The maximum swimming speed was realized by shortening the stroke cycle as much as possible although this shortening brought a lower propulsive efficiency as well as a lower stroke length. In a constant maximum joint torque condition, the swimmer does not push the water by turning the palm to the side in the latter half of the underwater stroke at the stroke cycle which brings the maximum swimming speed. The locus of the hand was relatively straight. At the slightly longer stroke cycle, the swimmer pushed the water until the end of the underwater stroke. The locus of the hand was still relatively straight. At the sufficiently longer stroke cycle, the swimmer also pushed the water until the end of the underwater stroke. The locus of the hand, however, became more curved and therefore became a so-called 'S-shaped' stroke.
\end{abstract}

Keywords : Swimming, Sports biomechanics, Sport engineering, Muscle and skelton, Biofluid dynamics, Optimization, Simulation, Crawl stroke

\section{Introduction}

The crawl is the fastest stroke among all strokes for humans. The main component of the thrust in the crawl is considered to be produced by the upper limbs, especially by the hands and forearms (Hollander et al., 1988). Therefore, the style of the arm stroke is extremely important in the crawl. For this reason, many studies related to the arm stroke have been conducted to date (Schleihauf et al., 1983, Schleihauf et al., 1988, Berger et al., 1995, Bixler and Riewald, 2002, Sato and Hino, 2003, Rouboa et al., 2006, Pai, 1987, Kudo et al., 2007, Sidelnik and Young, 2006, Nakashima and Takahashi, 2012a, Nakashima and Takahashi, 2012b, Takagi and Sanders, 2002, Matsuuchi et al., 2009). Most of these studies are summarized to analyze the swimming motions for existing athlete swimmers, or to estimate the characteristics of the fluid forces acting on the hands and forearms. Alternatively, as an attempt to solve computationally the 'optimal' stroke, Ito and Okuno (2003) and Ito (2007) solved the optimal stroke direction to maximize the thrust and efficiency from the lift/drag characteristics of the hand.

The optimal arm stroke should be solved taking the whole body motion of the swimmer into account, since the arm stroke is realized by the combined motion of the joints of the upper limbs and the complicated three-dimensional movement of the trunk. As a tool to treat the whole body dynamics in swimming, the swimming human simulation model SWUM (Nakashima et al., 2007, http://www.swum.org/) has been developed. Using SWUM, Nakashima and Sato (2009) conducted the optimizing calculation of the arm stroke in the crawl. In this pilot study, the genetic 
algorithm as an optimizing method was incorporated into SWUM, and the strokes which respectively maximize the swimming speed and propulsive efficiency were obtained. Nakashima et al. (2012) further extended this approach. In this previous study, an optimizing method, which consisted of the random search and the PSO (Particle Swarm Optimization) algorithm, was constructed. In order to consider the maximum joint torque characteristics of the swimmer as the constraint condition of the optimization, an experiment to measure the maximum joint torques was carried out for various joint angles and angular speeds. Using the measured experimental data as the reference values, a musculoskeletal simulation model was constructed. By the constructed musculoskeletal model, maximum joint torque characteristics in various conditions were investigated and used to create a database. Using this database, the optimizing calculation was finally conducted.

In the previous study, the maximum joint torque characteristics were fixed as a single constraint condition. Since this constraint condition was imposed as the upper limit of the joint torque in the optimizing calculation, the resultant arm stroke maximizing the swimming speed could be regarded as the 'maximal effort' swimming for the short distance event, such as $50 \mathrm{~m}$ or $100 \mathrm{~m}$. However, for longer distance events such as $200 \mathrm{~m}, 400 \mathrm{~m}, 800 \mathrm{~m}$ and $1500 \mathrm{~m}$, the competitive swimmers are considered not to use their maximal effort, but to reserve it. Such a situation is considered to be simulated by lowering the limit of the joint torque. Therefore, the optimizing calculation using the different lower maximum joint torque conditions should be conducted.

The objectives of this study were to solve computationally the arm stroke in the crawl swimming which maximized the swimming speed for different maximum joint torque conditions, and to investigate the maximum joint torque dependency of the crawl swimming with the optimized arm stroke. The simulation method, which was constructed in the previous study (Nakashima et al., 2012), is briefly described in $\S 2$. The results are discussed in $\S 3$. The main findings obtained in this study are summarized in $\S 4$.

\section{Simulation method}

\subsection{Outline of the swimming human simulation model SWUM}

In order to evaluate the arm strokes in the optimizing calculation, the swimming human simulation model SWUM was employed. SWUM was designed to solve the six degrees-of-freedom absolute movement of the whole swimmer's body as a single rigid body by time integration using the inputs of the swimmer's body geometry and relative joint motion. Therefore, the swimming speed, roll, pitch and yaw motions, propulsive efficiency, joint torques and so on, are computed as the output data. The swimmer's body is represented by a series of 21 rigid body segments as follows: lower and upper waist, lower and upper chest, shoulders, neck, head, upper and lower hips, thighs, shanks, feet, upper arms, forearms and hands. Each body segment is represented by a truncated elliptic cone. The unsteady fluid force and gravitational force are taken into account as external forces acting on the whole body. The unsteady fluid force is assumed to be the sum of the inertial force due to the added mass of the fluid, normal and tangential drag forces and buoyancy. These components are assumed to be computable, without solving the flow, from the local position, velocity, acceleration, direction, angular velocity, and angular acceleration for each part of the human body at each time step. The coefficients in this fluid force model were identified using the results of an experiment with a limb model and measurements of the drag acting on swimmers taking a glide position in the previous studies (Nakashima et al., 2007). As a result of the identification, the fluid force model was found to have satisfactory performance. Many other studies by SWUM, including its validation and application, have been already conducted to date (Nakashima, 2007, Nakashima, 2009, Kiuchi et al., 2010, Nakashima et al., 2010a, Nakashima et al., 2010b, Nakashima, 2010, Nakashima et al., 2013).

\subsection{Design variables and objective function}

In SWUM, the input swimming motion in one stroke cycle is represented as the time histories of the joint angle. In this study, one stroke was divided into 6 time frames for the upper limbs and 18 for the lower limbs. The joint angles between each of the two frames are automatically interpolated using the Spline function. The upper limb motion was represented by five degrees-of-freedom (DOF), that is, three at the shoulder joint and two at the elbow joint (flexion/extension and supination/pronation). Each of the five joint angles at three time frames, in which the arm is underwater, was chosen as the design variables. Therefore, the total number of design variables was 15 ( 5 DOF $\times 3$ frames). 
The objective function in the present study was the swimming speed, which was defined as the one averaged in a stroke cycle.

\subsection{Optimizing method}

In order to obtain the optimal solution as globally as possible within a reasonable computation time, two-step optimization was employed in this study. In the first step, the simple random search was used for global searching. In this search, 10,000 randomly generated arm strokes were evaluated and the best 20 were employed for the initial candidates of the second step. In the second step, the PSO (Particle Swarm Optimization) algorithm (Kennedy and Eberhart, 1995) was used for fine searching.

\subsection{Constraint conditions}

In the present study, three constraint conditions were imposed on the optimizing calculation. The first one related to the adduction angle of the shoulder joint. In the crawl, the adduction angle is considered to monotonously increase (the arm moves from head side to foot side). Therefore, when the three adduction angles for the three time frames were output from the optimizing algorithm, the order of these joint angles were rearranged so that the adduction angles monotonously increase. This constraint enabled the exclusion of arm strokes that were too unrealistic.

The second constraint was to prevent the penetration of the hand into the trunk of the swimmer. In order to prevent the penetration, a database to judge the penetration was constructed by calculating whether the hand penetrates the trunk or not for various joint angles. Using this database, the maximum elbow flexion angle could be calculated. If the elbow flexion angle output from the optimizing algorithm exceeded this limit, the flexion angle was forcibly reduced to the limit.

The third constraint related to the maximum joint torque characteristics of the swimmer. The details are described in the next subsection.

\subsection{Maximum joint torque characteristics}

Since the maximum joint torques are affected by the joint angles and angular speeds, they are not constant in the swimming stroke. Therefore, they were investigated and incorporated into the optimizing calculation in the present study as follows.

To begin with, an experiment using subject swimmers was conducted in order to experimentally acquire the maximum joint torques for various joint angles and angular speeds. The acquired experimental characteristics, however, were not sufficient for the optimization since the data were obtained only in limited experimental conditions. There were actually numerous combinations of angles and angular speeds of shoulder and elbow joints during the swimming stroke. In order to solve this problem, a musculoskeletal simulation in which the muscle activations were obtained for numerous combinations of joint angles, angular speeds, and torques was carried out. By using the information obtained by this simulation, it was enabled to calculate the maximum joint torque when a certain muscle was fully activated. Before the simulation, the musculoskeletal model had to be able to reproduce the experimental results. Therefore, the muscle parameters in the constructed musculoskeletal model were adjusted so that the simulation could reproduce the experimental characteristics. The details are described in the reference (Nakashima et al. 2012).

Using the adjusted muscle parameters, the maximum joint torques for various combinations of the angles and angular speeds of the shoulder and elbow joints were calculated by the simulation. The results were stored in the computer as a database, which was referred in the optimizing calculation. The reason for constructing the database was that the musculoskeletal simulation took too much computation time to be directly incorporated into the optimizing iteration. In the optimizing calculation, the maximum joint torques were calculated at each time step in SWUM by the interpolation of the database values. If a joint torque during swimming calculated by SWUM exceeded the maximum joint torque, a penalty term was added to the objective function so that the resultant objective function was reduced.

In order to investigate the maximum joint torque dependency of the optimized results, four levels of maximum joint torque (MJT), that is, 'MJT 1.0', 'MJT 0.9', 'MJT 0.8' and 'MJT 0.6' were prepared for the calculation. In MJT 0.6 , for example, all the values of the maximum joint torques in the database were simply multiplied by 0.6 . Therefore, it corresponds to the situation in which the swimmer only uses $60 \%$ of the maximal effort. 


\section{Results and discussion}

\subsection{Swimming speed, propulsive efficiency and stroke length}

The optimizing calculations were conducted in the condition that the stroke cycle was fixed to a constant value. It took 170 hours ( 7 days) to obtain the results for one optimizing calculation by a PC. The results of the swimming speeds are shown in Table 1. The ' $\mathrm{X}$ ' marks in the table means the cases where the solution could not be obtained. That is, all the 20 final candidates in the PSO algorithm largely violated the constraint condition with respect to the maximum joint torque. From this table, it was found that the shortest stroke cycle brought the maximum swimming speed in a constant maximum joint torque. The results of Table 1 are schematically plotted in Fig. 1. Note that some unevenness is seen in the line graphs because of the insufficient accuracy of the optimizing calculation. From Fig. 1, it was found that the swimming speed decreases according to the increase in the stroke cycle. It was also found that the swimming speed decreases according to the decrease in the maximum joint torque. Therefore, the stroke cycle producing the maximum swimming speed increases according to the decrease in the maximum joint torque. These results mean that the maximum swimming speed cannot be achieved in longer stroke cycles, that is, the swimmer has to choose the shortest stroke cycle to maximize his/her swimming speed. However, this does not necessarily mean that 'the shorter stroke cycle is better'. For example, if the swimmer has the maximum joint torque of MJT 0.6, the swimmer should choose the stroke cycle of $0.9 \mathrm{~s}$. If the swimmer chooses $0.7 \mathrm{~s}$, he/she cannot even swim, that is, the reasonable joint motion cannot be realized. Therefore, for the athletic swimmers, these results suggest the importance of choosing the appropriate stroke cycle for his/her maximum joint torque to enhance the performance.

The results of propulsive efficiency are shown in Fig. 2. In the present study, the propulsive efficiency $\eta$ is defined as

Table 1 Results of swimming speeds (unit is $\mathrm{m} / \mathrm{s}$ ).

\begin{tabular}{|c|cccc|}
\hline $\begin{array}{c}\text { Stroke } \\
\text { cycle [s] }\end{array}$ & MJT 1.0 & MJT 0.9 & MJT 0.8 & MJT 0.6 \\
\hline 0.5 & $\times$ & & & \\
0.6 & $\times$ & $\times$ & $\times$ & \\
0.7 & 2.03 & $\times$ & 1.81 & $\times$ \\
0.8 & 1.93 & 1.88 & 1.76 & 1.61 \\
0.9 & 1.99 & 1.87 & 1.76 & 1.55 \\
1.0 & 1.92 & 1.83 & 1.65 & 1.60 \\
1.1 & 1.83 & 1.74 & 1.72 & 1.51 \\
1.2 & 1.78 & 1.75 & 1.63 & 1.50 \\
1.3 & 1.68 & 1.66 & 1.58 & 1.45 \\
1.4 & 1.62 & 1.61 & 1.53 & 1.37 \\
1.5 & 1.56 & 1.57 & 1.40 & 1.36 \\
1.6 & & 1.39 & 1.81 & 1.61 \\
\hline max. & 2.03 & 1.88 & & \\
\hline
\end{tabular}

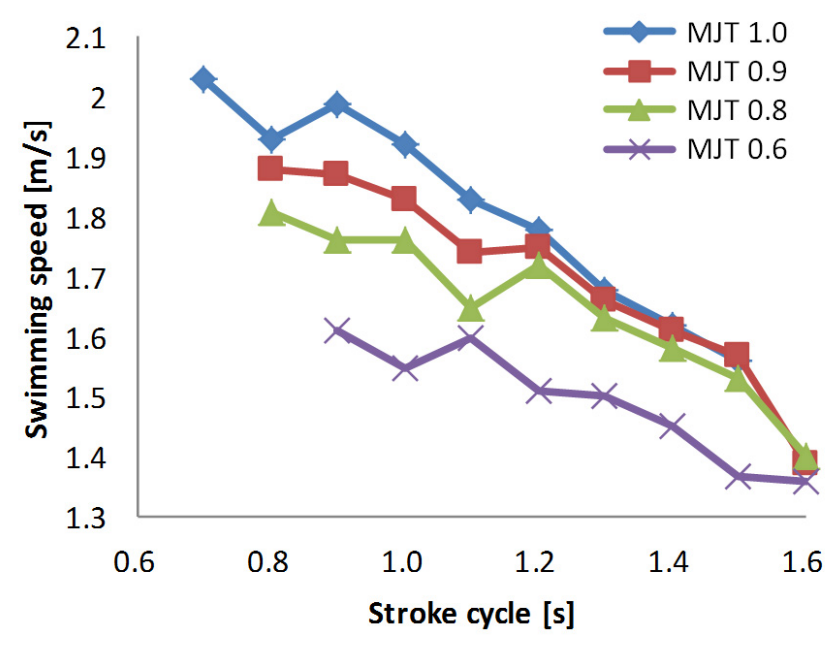

Fig. 1 Relationship between stroke cycle and swimming speed. 


$$
\eta=\frac{U D}{P}
$$

where $U, D$ and $P$ respectively represent the swimming speed $(\mathrm{m} / \mathrm{s})$, drag when the swimmer takes the gliding position $(\mathrm{N})$, and time-averaged power consumed by the swimmer $(\mathrm{W})$. The propulsive efficiency $\eta$ is nondimensional. The power consumed by the swimmer was calculated as the amount of local power which was calculated by the local external force acting on the swimmer (fluid force) multiplied by the local velocity at the local point in the swimmer's body. The joint passive resistance was not considered in the calculation since it was regarded as small. From Fig. 2, it was found that the propulsive efficiency decreased according to the decrease in the stroke cycle for all the maximum joint torque conditions. Therefore, it can be said that the maximum swimming speed was attained in the condition of the lowest propulsive efficiency. With respect to the maximum joint torque dependency, the propulsive efficiency decreased according to the decrease in the maximum joint torque, especially for the lower stroke cycles. However, since the stroke cycle which brought the maximum swimming speed increased according to the decrease in the maximum joint torque, the propulsive efficiency at the stroke cycle of the maximum swimming speed was mostly constant (around 0.15) for all the maximum joint torque conditions.

The results of the stroke length are shown in Fig. 3. The stroke length in the present study was defined as the propulsive distance in one stroke cycle, which was nondimensionalized by the swimmer's stature. From Fig. 3, it was found that the stroke length decreased according to the decrease in the stroke cycle and the maximum joint torque. As a

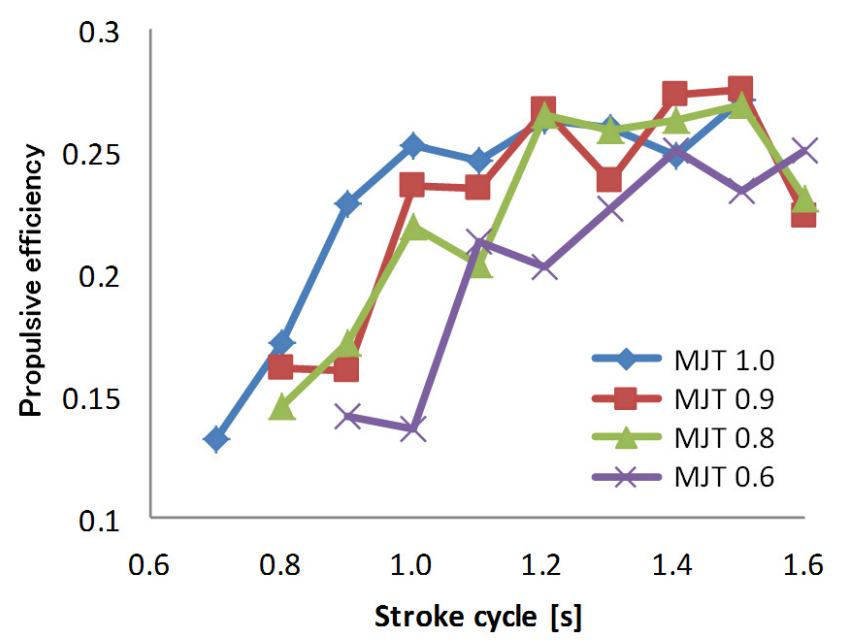

Fig. 2 Relationship between stroke cycle and propulsive efficiency.

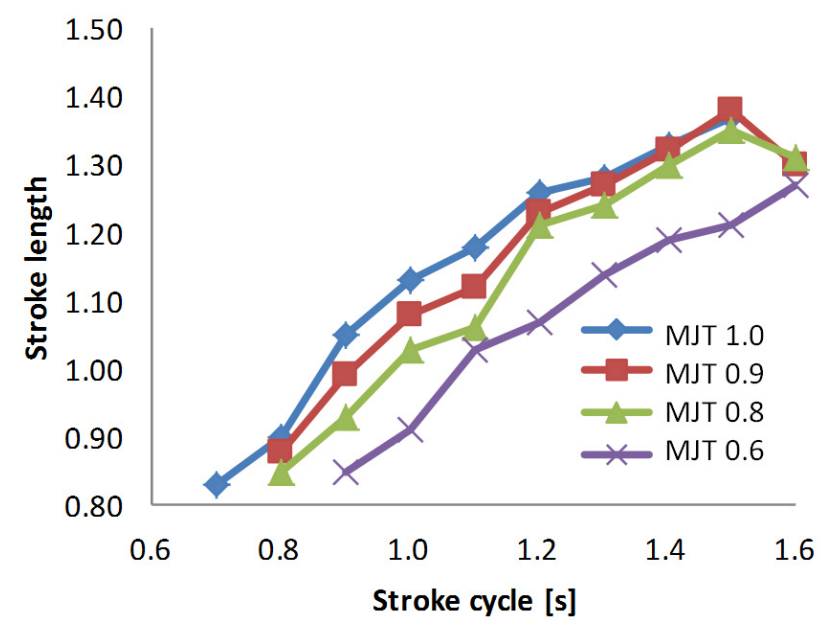

Fig. 3 Relationship between stroke cycle and stroke length. 
result, the stroke length at the stroke cycle of the maximum swimming speed was again mostly constant $(0.80 \sim 0.90)$ for all the maximum joint torque conditions.

To conclude this subsection, it was found that the maximum swimming speed was realized by shortening the stroke cycle as much as possible although this shortening brought the lower propulsive efficiency as well as the lower stroke length.

\subsection{Swimming motion}

As an example, the swimming motions and their characteristics for MJT 0.8 are schematically summarized in Fig. 4. In this figure, $t^{*}$ represents the nondimensional time, which is normalized by the stroke cycle. That is, one stroke cycle is $t^{*}=0.0$ to 1.0. Six moments are shown in the figure. The moments $t^{*}=0.0$ and 0.667 are the fixed frames (the joint angles were fixed for these frames in the optimization). The moments $t^{*}=0.167,0.333$ and 0.500 are the frames at which the joint angles were the design variables. The moment $t^{*}=0.440$ was used in order to show the difference of the palm directions. At the stroke cycle of $0.8 \mathrm{~s}$ (left column), which brought the maximum swimming speed, the swimmer did not push the water by turning the palm to the side in the latter half of the underwater stroke $\left(t^{*}=0.440\right)$. From the bottom view, it was found that the locus of the hand (shown in the red dotted line) was relatively straight. The reason why the palm was turned to the side in this calculation relates to the constraint condition of the design variables in the optimizing calculation. Since the adduction angle had to monotonously increase among $t^{*}=0.167,0.333,0.500$, the adduction angle at $t^{*}=0.500$ had to be larger than that at $t^{*}=0.333$. Therefore, it was not possible to end the underwater stroke earlier and raise the arm for the recovery in the present calculation. In the actual situation, it seems more reasonable and better to end the underwater stroke earlier and raise the arm for the recovery than to turn the palm

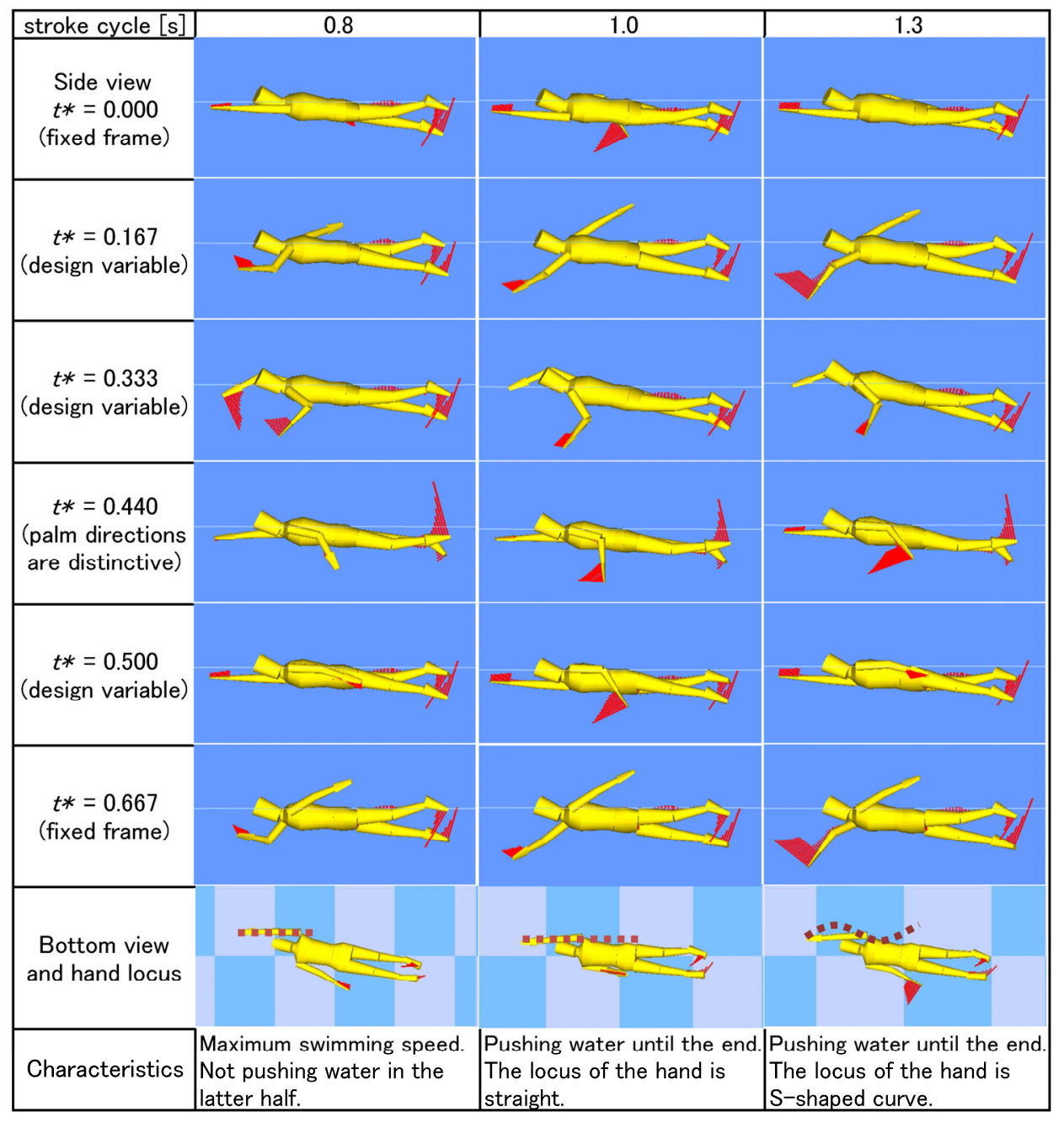

Fig. 4 Swimming motions and their characteristics for MJT 0.8. 


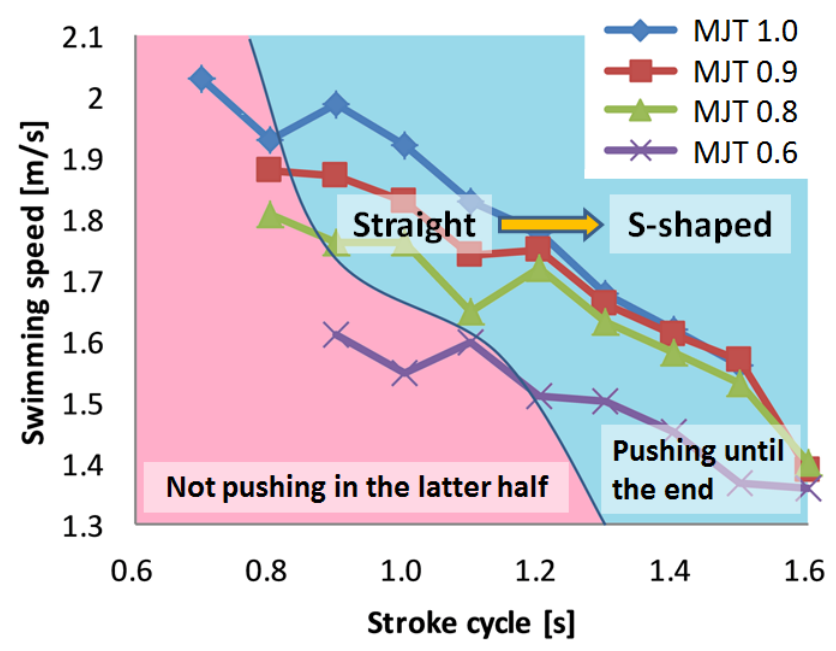

Fig. 5 Relationship between stroke cycle and swimming speed.

to the side. At the stroke cycle of 1.0s (center column), the swimmer pushed the water until the end of the underwater stroke. The locus of the hand was still relatively straight. At the stroke cycle of $1.3 \mathrm{~s}$ (right column), the swimmer also pushed the water until the end of the underwater stroke. The locus of the hand, however, became more curved and therefore became a so-called 'S-shaped' stroke. In addition, the stroke became a so-called 'high-elbow' at the catch phase $\left(t^{*}=0.167\right)$. Since the longer stroke cycle brought the higher propulsive efficiency as shown in Fig. 2 , it was suggested that the techniques of the 'high-elbow' and the 'S-shaped' stroke contributed to the increase in the propulsive efficiency. In the short stroke cycle, the swimmer could not take such techniques by the constraint of the maximum joint torque. However, by shortening the stroke cycle, that is, by increasing the number of the stroke in a constant time, the maximum swimming speed was attained.

Similar tendencies were also seen in the other maximum joint torque conditions. However, the values of the stroke cycle corresponding to the stroke style changed according to the change in the maximum joint torque. This is schematically explained in Fig. 5. For example, the 'not pushing in the latter half' stroke (the red area) was seen in the stroke cycles of $0.7 \mathrm{~s}$ and $0.8 \mathrm{~s}$ for MJT 1.0, while it was seen in the stroke cycles of $0.9 \mathrm{~s}, 1.0 \mathrm{~s}$ and 1.1s for MJT 0.6. In the 'pushing until the end' stroke (the blue area), the locus of the hand changed from straight (lower stroke cycle) to S-shaped (higher stroke cycle). Note that the swimming speed did not increase much according to the increase of the maximum joint torque for the stroke cycles higher than $1.2 \mathrm{~s}$. The reason for this might be the problem in the optimizing calculation. As shown in Fig. 4, the swimmer already finished the underwater stroke at the third frame for the design variable $\left(t^{*}=0.500\right)$ in the stroke cycle of $1.3 \mathrm{~s}$. That is, the swimmer finished the underwater stroke in the anterior part of the optimizing time period. Therefore, the underwater stroke was represented only by the two first time frames. This might not sufficient to form the good stroke which can produce the thrust efficiently. Therefore, there is a possibility for these stroke cycles that better strokes can be found by the improvement of the optimizing calculation.

\section{Conclusions}

In this study, the arm strokes in the crawl swimming which maximized the swimming speed for different maximum joint torque conditions were solved computationally. The maximum joint torque dependency of the crawl swimming with the optimized arm stroke was investigated. The main findings are summarized as follows:

( 1 ) The shortest stroke cycle brought the maximum swimming speed in a constant maximum joint torque.

( 2 ) The stroke cycle producing the maximum swimming speed increased according to the decrease in the maximum joint torque.

( 3 ) The maximum swimming speed was realized by shortening the stroke cycle as much as possible although this shortening brought the lower propulsive efficiency as well as the lower stroke length.

( 4 ) In a constant maximum joint torque condition, the swimmer does not push the water by turning the palm to the side in the latter half of the underwater stroke at the stroke cycle which brings the maximum swimming speed. The locus of the hand was relatively straight. At the slightly longer stroke cycle, the swimmer pushed the water until 
the end of the underwater stroke. The locus of the hand was still relatively straight. At the sufficiently longer stroke cycle, the swimmer also pushed the water until the end of the underwater stroke. The locus of the hand, however, became more curved and therefore became a so-called 'S-shaped' stroke.

\section{References}

Berger, M.A.M.,de Groot, G. and Hollander A.P., Hydrodynamic drag and lift forces on human hand/arm models, Journal of Biomechanics, Vol.28, (1995), pp.125-133.

Bixler, B. and Riewald, S., Analysis of a swimmer's hand and arm in steady flow conditions using computational fluid dynamics, Journal of Biomechanics, Vol.35, (2002), pp.713-717.

Hollander, A.P., de Groot, G. and van Ingen Schenau, G.J., Contribution of the legs to propulsion in front crawl swimming, Swimming Science V, (1988), pp.17-29.

Ito, S., Optimal Armstroke for swimming of freestyle, Transactions of the Japan Society of Mechanical Engineers, Series B, Vol.73, No.734, (2007), pp.2051-2057 (in Japanese).

Ito, S. and Okuno, K., A Fluid dynamical consideration for arm stroke in swimming, Biomechanics and Medicine in Swimming IX, (2003), pp.39-44.

Kennedy, J. and Eberhart, R.C., Particle swarm optimization, Proceedings of 1995 IEEE International Conference on Neural Networks, Vol.4, (1995), pp.1942-1948.

Kiuchi, H., Nakashima, M., Cheng, K.B. and Hubbard, M. Modeling fluid forces in the dive start of competitive swimming, Journal of Biomechanical Science and Engineering, Vol.5, No.4, (2010), pp.314-328.

Kudo, S., Wilson, B., and Takagi, H., The error of a quasi-static approach in predicting fluid Forces on the hand in unsteady conditions, Japanese Journal of Sciences in Swimming and Water Exercise, Vol.10, No.1, (2007), pp.1-11.

Matsuuchi, K., Miwa, T., Nomura, T., Sakakibara, J., Shintani, H. and Ungerechts, B.E., Unsteady flow field around a human hand and propulsive force in swimming, Journal of Biomechanics, Vol.42, (2009), pp.42-47.

Nakashima, M., Analysis of breast, back, and butterfly strokes by the swimming human simulation model SWUM, Bio-mechanisms of Swimming and Flying - Fluid Dynamics, Biomimetic Robots, and Sports Science - (eds. Naomi Kato and Shinji Kamimura), Springer, Tokyo, (2007), pp.361-372.

Nakashima, M., Simulation analysis of the effect of trunk undulation on swimming performance in underwater dolphin kick of human, Journal of Biomechanical Science and Engineering, Vol. 4, No. 1, (2009), pp.94-104.

Nakashima, M. Modeling and simulation of human swimming, Journal of Aero Aqua Bio-mechanisms, Vol.1, No.1, (2010), pp.11-17.

Nakashima, M., Hatakeyama, G., Homma, M. and Ito, K., Simulation analysis of lift in synchronized swimming, Journal of Aero Aqua Bio-mechanisms, Vol.3, No.1, (2013), pp.51-56.

Nakashima, M., Kiuchi, H. and Nakajima, K. Multi agent/object simulation in human swimming, Journal of Biomechanical Science and Engineering, Vol. 5, No. 4, (2010), pp.380-387.

Nakashima, M., Maeda, S., Miwa, T. and Ichikawa, H., Optimizing simulation of the arm stroke in crawl swimming considering muscle strength characteristics of athlete swimmers, Journal of Biomechanical Science and Engineering, Vol.7, No.2 (2012), pp.102-117.

Nakashima, M., Miura, Y. and Satou, K. Development of swimming human simulation model considering rigid body dynamics and unsteady fluid force for whole body, Journal of Fluid Science and Technology, Vol. 2, No. 1, (2007), pp.56-67.

Nakashima, M. and Sato, Y., Optimization of arm stroke in freestyle swimming by simulation, The Impact of Technology on Sport III (Proceedings of the Asia-Pacific Congress on Sport Technology), (2009), pp.207-211.

Nakashima, M., Suzuki, S. and Nakajima, K. Development of a simulation model for monofin swimming, Journal of Biomechanical Science and Engineering, Vol. 5, No. 4, (2010), pp.408-420.

Nakashima, M. and Takahashi, A., Clarification of unsteady fluid forces acting on limbs in swimming using an underwater robot arm (development of an underwater robot arm and measurement of fluid forces), Journal of Fluid Science and Technology, Vol.7, No.1, (2012a), pp.100-113.

Nakashima, M. and Takahashi, A., Clarification of unsteady fluid forces acting on limbs in swimming using an underwater robot arm (2nd report, modeling of fluid force using experimental results), Journal of Fluid Science 
and Technology, Vol.7, No.1, (2012b), pp.114-128.

Pai, Y., A Hydrodynamic Study of the oscillation motion in swimming, Unpublished PhD thesis, (1987), The University of Iowa.

Rouboa, A., Silva, A., Leal, L., Rocha, J. and Alves, F., The effect of swimmer's hand/forearm acceleration on propulsive forces generation using computational fluid dynamics, Journal of Biomechanics, Vol.39 (2006), pp.1239-1248.

Sato, Y. and Hino, T., Estimation of thrust of swimmer's hand using CFD, Proceedings of the Second International Symposium on Aqua Bio-mechanisms, (2003), P.2-07 (CDROM).

Schleihauf, R.E., Gray, L., and DeRose J., Three-dimensional analysis of hand propulsion in the sprint front crawl stroke, Biomechanics and Medicine in Swimming IV, (1983), pp.173-184.

Schleihauf, R.E., Higgins, J.R., Hinrichs, R., Luedtke, D., Maglischo, E.W. and Thayer, A., Propulsive techniques: front crawl stroke, butterfly, backstroke, and breaststroke, Swimming Science V, (1988), pp.53-59.

Sidelnik, N.O. and Young, B.W., Optimising The freestyle swimming stroke: the effect of finger spread, Sports Engineering, Vol.9, (2006), pp.129-135.

Takagi, H. and Sanders, R., Measurement of propulsion by the hand during competitive swimming, The Engineering of Sport 4 (Eds. Ujihashi, S and Haake, S.J.), (2002), pp.631-637, Blackwell Publishing. 